\title{
Essential literature for the chiropractic profession: a survey of chiropractic research leaders
}

\author{
Barbara A Mansholt ${ }^{*}$, John S Stites ${ }^{2}$, Dustin C Derby ${ }^{3}$, Ron J Boesch ${ }^{4}$ and Stacie A Salsbury ${ }^{5}$
}

\begin{abstract}
Background: Evidence-based clinical practice (EBCP) is an accepted practice for informed clinical decision making in mainstream health care professions. EBCP augments clinical experience and can have far reaching effects in education, policy, reimbursement and clinical management. The proliferation of published research can be overwhelming - finding a mechanism to identify literature that is essential for practitioners and students is desirable. The purpose of this study was to survey leaders in the chiropractic profession on their opinions of essential literature for doctors of chiropractic, faculty, and students to read or reference.

Methods: Deployment of an IRB exempted survey occurred with 68 academic and research leaders using SurveyMonkey ${ }^{\oplus}$. Individuals were solicited via e-mail in August of 2011; the study closed in October of 2011. Collected data were checked for citation accuracy and compiled to determine multiple responses. A secondary analysis assessed the scholarly impact and Internet accessibility of the recommended literature.

Results: Forty-three (43) individuals consented to participate; seventeen (17) contributed at least one article of importance. A total of 41 unique articles were reported. Of the six articles contributed more than once, one article was reported 6 times, and 5 were reported twice.

Conclusions: A manageable list of relevant literature was created. Shortcomings of methods were identified, and improvements for continued implementation are suggested. A wide variety of articles were reported as "essential" knowledge; annual or bi-annual surveys would be helpful for the profession.
\end{abstract}

Keywords: Evidence-based practice, Evidence-based health care, Chiropractic, Web-based survey, Publications, Professional education

\section{Background}

Evidence based clinical practice (EBCP) may be defined as "the conscientious and judicious use of clinical expertise, patient values, and current best evidence" [1]. Each component of EBCP may assume greater or lesser importance depending upon such factors as the doctor-patient relationship or the patient's presenting condition and preferences, and assert varying emphases within different healthcare environments or health service delivery contexts [2] (Figure 1). While definitions may vary, evidence within an EBCP framework refers to published, peerreviewed, scientific research [1]. Thus, EBCP requires that clinicians are knowledgeable of current scientific evidence and best practices based upon published research [3].

\footnotetext{
* Correspondence: barbara.mansholt@palmer.edu

${ }^{1}$ Technique Department, Palmer College of Chiropractic, 1000 Brady St, Davenport, IA 52803, USA

Full list of author information is available at the end of the article
}

The chiropractic profession has maintained its position as the largest alternative or non-traditional approach to healthcare [4-6]. At the same time, the profession seeks wider mainstream acceptance by both the public and its healthcare peers [7-16]. In response, many chiropractic colleges now teach EBCP approaches to chiropractic care [17-20]. Used appropriately, EBCP gives chiropractic professionals of all stages of experience insight beyond their own clinical expertise to guide clinical decision making and helps patients make educated, informed choices [21]. However, recent studies suggest chiropractors and chiropractic students may not yet possess the attitudes, knowledge, reading habits or critical appraisal skills needed to implement EBCP in educational or practice settings [19,22-24].

One issue in EBCP consistently identified by chiropractic students and clinicians is the process of searching,

\section{Biomed Central}




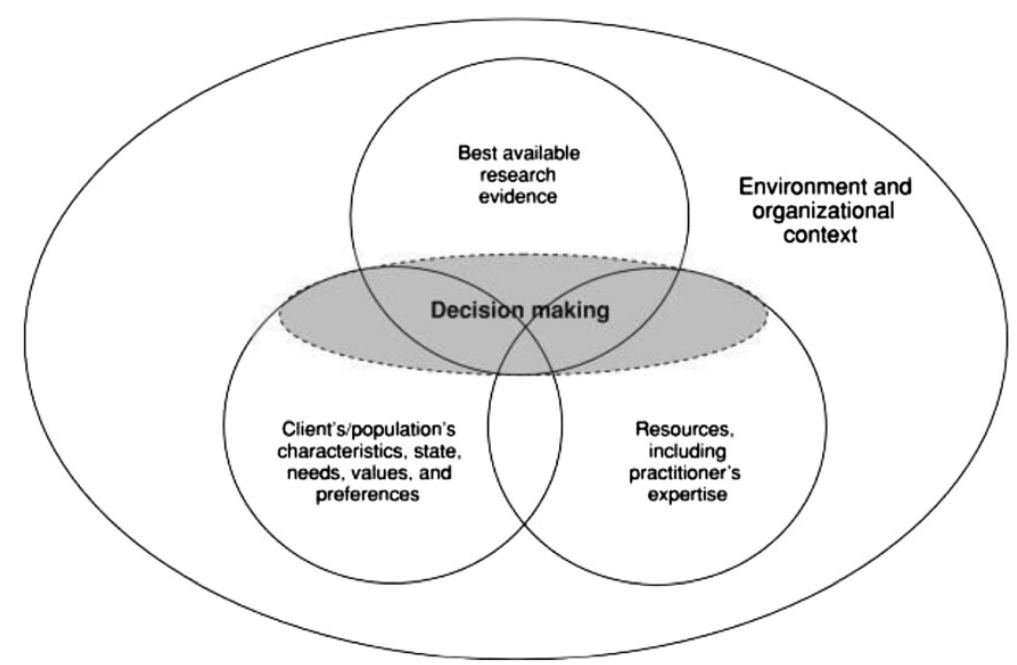

Figure 1 Evidence based clinical decision making Venn diagram².

reading, and appraising the scientific literature to apply this evidence to clinical practice [22-26]. At the same time, published research in the chiropractic profession is increasing in quality and quantity at a rapid pace [27-30]. Clinicians in all stages of practice might benefit from compiled resources that identify key studies and other evidence to support chiropractic practice. Chiropractic researchers frequently review and appraise the scholarly literature related to the field of chiropractic and might provide input into which articles would be most important to doctors of chiropractic [28]. Our goal in this study was to compile a list of the most important published research germane to the field of chiropractic-articles every doctor of chiropractic, students, or faculty should read, reference, and put into EBCP.

\section{Methods}

The Palmer College of Chiropractic Institutional Review Board (IRB) Human Protections Administrator exempted this survey from full IRB review, and the study was approved as submitted. The authors compiled a list of potential survey respondents that included the research department directors at 18 chiropractic colleges in North America, editors of 17 chiropractic journals, and respected leaders within the profession based upon their current involvement in research and/or regard as a prominent research author. We identified an initial list of 68 chiropractic research leaders.

We developed a web-based survey using Survey Monkey ${ }^{\circ}$ software and pre-tested it among the authors. Recipients received the survey link via e-mail and cover letter with a short explanation regarding the purpose and methods of the study. Upon linking to the survey, recipients were informed that no personal benefit would be gained through their response and that their response was anonymous. Checking a box "yes" to advance into the survey served as informed consent and was stated as such in the dialogue box. Survey deployment occurred between August and October 2011. Respondents received up to four automated reminder messages, depending on their response status within the software. Demographic data collected included respondents' age, gender, highest degree earned, primary academic assignment (e.g., research, faculty, clinician, administration), and area of focus.

The survey used qualitative data collection methods as presenting respondents with an exhaustive list of research studies germane to the field of chiropractic in a checklist format was not feasible. Instead, respondents listed the author, title, journal, and year of an article the respondent considered important and one "that every doctor of chiropractic should read". Respondents then categorized the article as education, research, health care policy, patient education, or other. Each respondent also wrote a short statement indicating why the recommended article was important for the field. Each respondent could list up to six research studies. Quality control checks assured citation accuracy. Citation recommendations were analyzed thematically according to Bogdan and Biklen's "subjects" ways of thinking about people and objects" [31]. In other words, we were interested in knowing which objects (e.g., published research) the respondent believed were the most critical for chiropractic practitioners and students (e.g., their thoughts on importance).

We performed a secondary analysis to characterize the scholarly impact and accessibility of each recommended article to assist chiropractic professionals in the process of selecting high-quality evidence from among the scholarly literature. Scholarly impact refers to the relative importance of a publication within its field and across disciplines. Google Scholar was selected as the literature 
search engine over alternate scientific and academic literature repositories (e.g., PubMed, Index of Chiropractic Literature, Scopus, Web of Science) for its no-cost public access, breadth of coverage across disciplines, ability to search multiple databases simultaneously, embedded links to recent and full-text articles, and literature citation metrics. We estimated scholarly impact by counts of the number of times other researchers had cited each article.

Practitioners' access to scholarly literature is a known barrier to implementation of EBCP across disciplines with many clinicians reporting little to no access to electronic resources in their workplaces [25,32-34]. Article accessibility was assessed through the 'All Versions' feature of Google Scholar. We characterized each article as fully accessible if it was available without cost through open access sources (e.g., PubMed Central [PMC] or the journal/publisher website), limitedly accessible if an article required purchase from the publisher for on-line access, and inaccessible if no link to an on-line source or purchase options was identified.

\section{Statement on ethics}

This study was submitted to the Palmer College of Chiropractic Human Protections Administrator in June of 2011, determined exempt according to 45 CFR 46.101 (b)(2), and conducted from August of 2011 through October or 2011. A signed copy of IRB assurance \# X2011-2-1-S is available upon request.

\section{Results}

Response rates and respondent demographics

Although 43 of 68 potential respondents agreed to participate in the study (63\%), only 17 respondents contributed at least one article of importance, for a response rate of $25 \%$. Of the final respondents, six were between 41-50 years of age, ten were between 51 and 60 , and one was 61 or older. In addition to possessing a clinical doctorate, nine held academic doctorates, six master's degrees, and twelve bachelor's degrees. Demographic characteristics of survey respondents are presented in Table 1.

\section{Article citation data}

Respondents contributed 50 pieces of evidence (PE). Forty-one PE were mentioned at least once, with six articles mentioned by two or more respondents. Respondents categorized 26 as research, six as education, six as health care policy, and the remaining three as other. Additionally, forty were published within journals and one was a book. Recommended articles were published most often in the Journal of Manipulative and Physiological Therapeutics and The Spine Journal (Figure 2). Publications years for the recommended PE ranged from
Table 1 Respondent demographics of essential literature study

\begin{tabular}{|c|c|}
\hline Characteristic & $\mathrm{n}=17$ \\
\hline \multicolumn{2}{|l|}{ Age } \\
\hline $41-50$ & 6 \\
\hline $51-60$ & 10 \\
\hline 61 or older & 1 \\
\hline \multicolumn{2}{|l|}{ Gender } \\
\hline Female & 1 \\
\hline Male & 16 \\
\hline \multicolumn{2}{|l|}{ Degrees earned (mark all that apply) } \\
\hline BS & 12 \\
\hline MS & 6 \\
\hline Clinical doctorate (DC, DO, MD) & 17 \\
\hline Academic doctorate (PhD or EdD) & 9 \\
\hline \multicolumn{2}{|c|}{ Primary involvement in chiropractic profession } \\
\hline Research & 8 \\
\hline Teaching (full-time) & 2 \\
\hline Faculty Clinician & 1 \\
\hline Practicing clinician (non-academic) & 3 \\
\hline Administration & 2 \\
\hline Other & 1 \\
\hline \multicolumn{2}{|l|}{ Area of focus } \\
\hline Health care policy & 1 \\
\hline Patient care & 2 \\
\hline Education & 3 \\
\hline Research & 9 \\
\hline Other & 2 \\
\hline
\end{tabular}

1995 to 2011, with an emphasis on recently published journal articles. Seventeen (17) PE were published in 2005-2009 and 11 PE between 2010-2011 (Figure 3).

Table 2 reports the 41 recommended PE. Citations are listed first by the number of recommendations by survey respondents and then by number of Google Scholar citations. Each PE also includes information about its accessibility. Lastly, a quotation from the recommending respondent is provided demonstrating the importance of the article for chiropractic professionals.

\section{Articles with multiple recommendations}

Six distinct articles were recommended by more than one respondent. Six respondents recommended the article, "Effectiveness of manual therapies: the UK evidence report", by Bronfort et al. [35]. A majority of the respondents noted the comprehensive nature of this systematic review of randomized clinical trials of spinal manipulation, mobilization and massage. This article addresses varying levels of evidence and cites 322 articles on spinal 


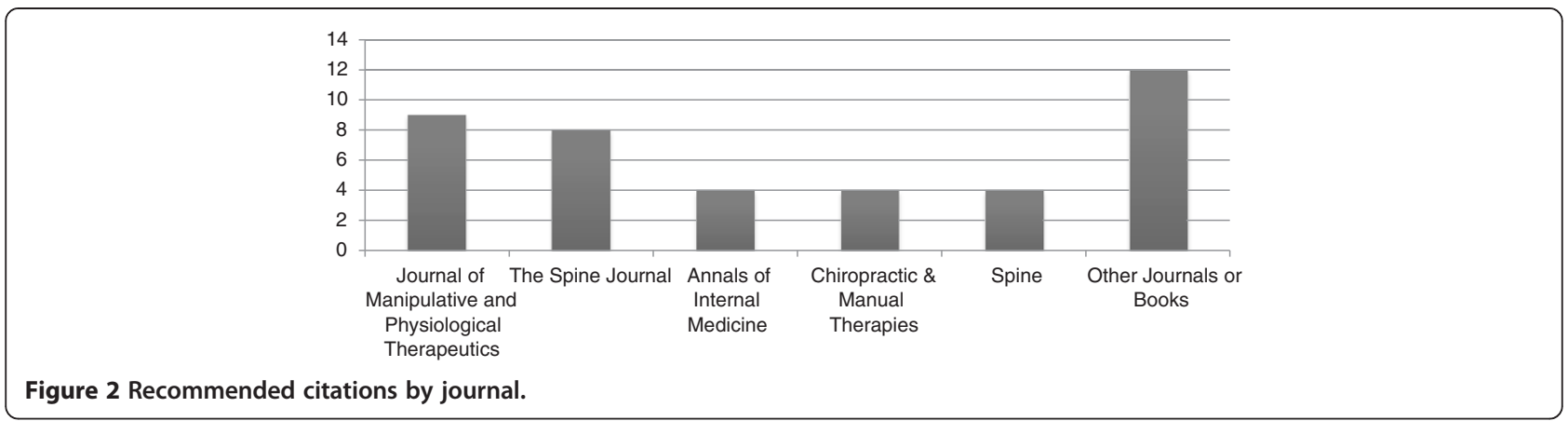

manipulation and other manual therapies as treatments for musculoskeletal, headache and non-musculoskeletal conditions in an organized and easily readable manner. One respondent wrote, "This is the most comprehensive systematic review of the literature pertinent to DCs for both musculoskeletal (MSK) and non-MSK conditions".

Two respondents each recommended five additional studies [8,36-39]. An article published in 2002 by Pickar [36], "Neurophysiological effects of spinal manipulation", is one of the earliest publications making the list, confirming the author's early attention to identifying the basic mechanisms of action underlying spinal manipulation and the relevance of the topic for doctors of chiropractic. One respondent wrote, "[the article] provides evidence based scientific rationale for the effects of spinal manipulation".

Another basic science article, this one by Ianuzzi and Khalsa [39], "Comparison of human lumbar facet joint capsule strains during simulated high-velocity, lowamplitude spinal manipulation versus physiological motions," was similarly recommended by respondents: "an important paper for mechanisms of action of spinal manipulation" and "demonstrated intrinsic biomechanical safety of lumbar spinal manipulation".

The large population-based study by Cassidy et al. [37] "Risk of vertebrobasilar stroke and chiropractic care: results of a population-based case-control and casecrossover study", demonstrated vertebrobasilar stroke as a very rare adverse event. This article is a relevant reference for chiropractic professionals who educate their patients, other healthcare providers and the media about the risks of chiropractic care. One respondent commented, "This presents the best evidence about the risk of stroke after cervical manipulation".

A Cochrane Collaboration systematic review published by Walker and colleagues [38], "Combined chiropractic interventions for low back pain", represented a rigorous, international, and interdisciplinary evaluation of the merits combined chiropractic therapies over spinal manipulation alone for pain and disability. Of this article, one respondent wrote, "This systematic review shows that usual chiropractic care is as effective as many other therapies for acute or sub acute low back pain".

An article by Murphy et al. [8], "The establishment of a primary spine care practitioner and its benefits to health care reform in the United States," presented a cogent health policy rationale for changing the role of chiropractors in the U.S. health care delivery system based on research evidence. Individuals interested in the evolution of the chiropractic profession should be familiar with this document, as a respondent commented, "This article outlines the need for a primary spine care clinician in the health care system, and highlights the qualifications of DCs to fill this niche".

\section{Literature with single recommendations}

Of the 41 unique PEs, 34 were unduplicated articles or books mentioned by a single respondent. Six singly recommended articles address interdisciplinary clinical

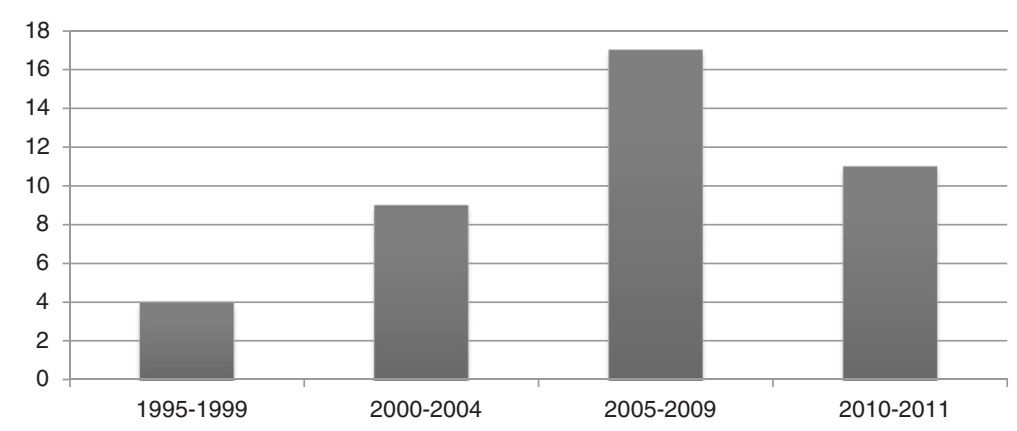

Figure 3 Recommended citations by publication year. 
Table 2 Essential literature for the chiropractic profession: articles submitted, number of recommendations and justification, Google scholar citation frequency, and accessibility

\begin{tabular}{|c|c|c|c|c|}
\hline Citation & $\begin{array}{c}\text { Survey } \\
\text { votes }\end{array}$ & $\begin{array}{c}\text { Google } \\
\text { scholar citations }\end{array}$ & $\begin{array}{l}\text { Open access } \\
\text { available }\end{array}$ & Recommendation quote \\
\hline $\begin{array}{l}\text { Bronfort } \mathrm{G}, \\
\text { et al [35] }\end{array}$ & 6 & 134 & PMC & $\begin{array}{l}\text { "This is the most comprehensive systematic review of the literature pertinent to DCs for both } \\
\text { musculoskeletal (MSK) and non-MSK conditions." }\end{array}$ \\
\hline Pickar JG [36] & 2 & 183 & $\begin{array}{l}\text { Purchase } \\
\text { Un/author }\end{array}$ & "provides evidence based scientific rationale for the effects of spinal manipulation." \\
\hline $\begin{array}{l}\text { Cassidy JD, } \\
\text { et al [37] }\end{array}$ & 2 & 174 & PMC & "This presents the best evidence about the risk of stroke after cervical manipulation." \\
\hline $\begin{array}{l}\text { Walker BF, } \\
\text { et al [38] }\end{array}$ & 2 & 32 & Purchase & $\begin{array}{l}\text { "This systematic review shows that usual chiropractic care is as effective as many other } \\
\text { therapies for acute or sub acute low back pain." }\end{array}$ \\
\hline $\begin{array}{l}\text { lanuzzi A, } \\
\text { et al [39] }\end{array}$ & 2 & 24 & PMC & $\begin{array}{l}\text { "An important paper for mechanisms of action of spinal manipulation." "Demonstrated } \\
\text { intrinsic biomechanical safety of lumbar spinal manipulation." }\end{array}$ \\
\hline $\begin{array}{l}\text { Murphy DR, } \\
\text { et al [8] }\end{array}$ & 2 & 6 & Journal & $\begin{array}{l}\text { "This article outlines the need for a primary spine care clinician in the health care system, } \\
\text { and highlights the qualifications of DCs to fill this niche." }\end{array}$ \\
\hline $\begin{array}{l}\text { Chou R, } \\
\text { et al }[40]\end{array}$ & 1 & 753 & Journal & $\begin{array}{l}\text { "Excellent summary of } 7 \text { recommendations related to the diagnosis and treatment of low } \\
\text { back pain." }\end{array}$ \\
\hline $\begin{array}{l}\text { Childs JD, } \\
\text { et al [41] }\end{array}$ & 1 & 435 & Journal & $\begin{array}{l}\text { "...published by PTs and is cited as the first clinical prediction rule (CPR) for spinal } \\
\text { manipulation. Although as DCs we feel "underwhelmed" by the simplistic nature of this CPR, } \\
\text { we must recognize that this paper has catapulted the PT profession's image within the } \\
\text { spine care world." }\end{array}$ \\
\hline $\begin{array}{l}\text { Chou } R, \\
\text { et al }[42]\end{array}$ & 1 & 389 & Journal & $\begin{array}{l}\text { "linical practice guidelines for primary care physicians for treatment of low back pain that } \\
\text { includes spinal manipulation" }\end{array}$ \\
\hline $\begin{array}{l}\text { UK BEAM Trial } \\
\text { Team [43] }\end{array}$ & 1 & 245 & PMC & "largest effectiveness trial to date of benefits of spinal manipulation for low back pain" \\
\hline $\begin{array}{l}\text { Senstad O, } \\
\text { et al [44] }\end{array}$ & 1 & 209 & Purchase & $\begin{array}{l}\text { "Chiropractors need to know that there are side effects to SMT and that not all patients } \\
\text { have a positive experience or outcome." }\end{array}$ \\
\hline $\begin{array}{l}\text { Hurwitz EL, } \\
\text { et al [45] }\end{array}$ & 1 & 207 & $\begin{array}{l}\text { Purchase } \\
\text { Un/author }\end{array}$ & $\begin{array}{l}\text { "The authors assess the evidence base for spinal manipulation and other conservative } \\
\text { therapies for the treatment of neck-related disorders." }\end{array}$ \\
\hline $\begin{array}{l}\text { Nelson CF, } \\
\text { et al }[46]\end{array}$ & 1 & 154 & Purchase & $\begin{array}{l}\text { "showed long term benefits of manipulation over most commonly used medication for } \\
\text { migraine headaches." }\end{array}$ \\
\hline $\begin{array}{l}\text { Olafsdottir E, } \\
\text { et al [47] }\end{array}$ & 1 & 141 & Journal & $\begin{array}{l}\text { "This paper demonstrates that the benefit of chiropractic for infantile colic is the same as a } \\
\text { placebo." }\end{array}$ \\
\hline $\begin{array}{l}\text { Skyba DA, } \\
\text { et al [48] }\end{array}$ & 1 & 88 & PMC & $\begin{array}{l}\text { "This paper presents the best neurophysiological evidence regarding post manipulative } \\
\text { analgesia." }\end{array}$ \\
\hline $\begin{array}{l}\text { Haldeman } \mathrm{S} \text {, } \\
\text { et al [49] }\end{array}$ & 1 & 79 & PMC & $\begin{array}{l}\text { "good summary of status of neck pain research relevant to chiropractors; would } \\
\text { recommend all papers in that series" }\end{array}$ \\
\hline $\begin{array}{l}\text { Dagenais } S \text {, } \\
\text { et al [50] }\end{array}$ & 1 & 76 & $\begin{array}{l}\text { Purchase } \\
\text { Un/author }\end{array}$ & "important to understand the current evidence and its implication for practice." \\
\hline $\begin{array}{l}\text { Legorreta AP, } \\
\text { et al [51] }\end{array}$ & 1 & 59 & Journal & "excellent paper assessing cost benefits to chiropractic care" \\
\hline $\begin{array}{l}\text { Cramer GD, } \\
\text { et al [52] }\end{array}$ & 1 & 56 & $\begin{array}{l}\text { Purchase } \\
\text { Un/author }\end{array}$ & "mechanisms of action of spinal manipulation" \\
\hline $\begin{array}{l}\text { Haas } M \text {, } \\
\text { et al [53] }\end{array}$ & 1 & 50 & $\begin{array}{l}\text { Purchase } \\
\text { Un/author }\end{array}$ & $\begin{array}{l}\text { "Dose studies of manipulation are extremely important. This one showing that more care } \\
\text { [is] better than less..." }\end{array}$ \\
\hline $\begin{array}{l}\text { Carroll LU, } \\
\text { et al [54] }\end{array}$ & 1 & 44 & PMC & $\begin{array}{l}\text { "The authors assess the evidence base for spinal manipulation and other conservative } \\
\text { therapies for the treatment of neck-related disorders." }\end{array}$ \\
\hline $\begin{array}{l}\text { Bogduk N, } \\
\text { et al [55] }\end{array}$ & 1 & 37 & $\begin{array}{l}\text { No online } \\
\text { access }\end{array}$ & $\begin{array}{l}\text { "This would give chiropractors a clear insight into and synthesis of the evidence } \\
\text { available for the treatment and management of neck pain" }\end{array}$ \\
\hline $\begin{array}{l}\text { Pickar JG, } \\
\text { et al [56] }\end{array}$ & 1 & 37 & Purchase & "important paper for mechanism of action of spinal manipulation" \\
\hline $\begin{array}{l}\text { Haas } M \text {, } \\
\text { et al [57] }\end{array}$ & 1 & 34 & $\begin{array}{l}\text { Purchase } \\
\text { Un/author }\end{array}$ & $\begin{array}{l}\text { "provides evidence that the frequency of effective manipulative care may not be what is } \\
\text { generally thought in clinical practice" }\end{array}$ \\
\hline Reggars JW, & 1 & 33 & Purchase & "it demonstrates which joints cavitate in the neck when the head is rotated left or right." \\
\hline
\end{tabular}


Table 2 Essential literature for the chiropractic profession: articles submitted, number of recommendations and justification, Google scholar citation frequency, and accessibility (Continued)

\begin{tabular}{|c|c|c|c|c|}
\hline $\begin{array}{l}\text { Murphy DR, } \\
\text { et al [59] }\end{array}$ & 1 & 32 & Journal & $\begin{array}{l}\text { "This paper is the first in a series to present a rationale for and the details of a rational } \\
\text { evidence based approach to the management of patients with spinal pain." }\end{array}$ \\
\hline $\begin{array}{l}\text { Bishop PB, } \\
\text { et al }[60]\end{array}$ & 1 & 27 & $\begin{array}{l}\text { Purchase } \\
\text { Un/author }\end{array}$ & "demonstrates practical application of EBM to clinical care" \\
\hline $\begin{array}{l}\text { Little JS, } \\
\text { et al }[61]\end{array}$ & 1 & 26 & Purchase & $\begin{array}{l}\text { "demonstrated that vertebral motion unit fixation (aka subluxation) creates abnormal facet } \\
\text { joint capsule strain patterns" }\end{array}$ \\
\hline $\begin{array}{l}\text { Pickar JG, } \\
\text { et al [62] }\end{array}$ & 1 & 23 & PMC & "demonstrated [that] spinal manipulation induces paraspinal muscle afferent response" \\
\hline Herzog W [63] & 1 & 19 & $\begin{array}{l}\text { Purchase } \\
\text { Un/author }\end{array}$ & "reviews the current evidence related to adjusting" \\
\hline $\begin{array}{l}\text { Haas } M \text {, } \\
\text { et al }[30]\end{array}$ & 1 & 18 & Purchase & "provides a historical summary" \\
\hline $\begin{array}{l}\text { Murphy DR, } \\
\text { et al [59] }\end{array}$ & 1 & 15 & Journal & $\begin{array}{l}\text { "2nd in the series by Murphy et al. that presents the evidence for the model presented earlier } \\
\text { on a chiropractic management approach to spinal pain" }\end{array}$ \\
\hline $\begin{array}{l}\text { lanuzzi A, } \\
\text { et al [64] }\end{array}$ & 1 & 14 & Purchase & "determined unique biomechanics created by spinal manipulation" \\
\hline $\begin{array}{l}\text { Hartman SE } \\
{[65]}\end{array}$ & 1 & 13 & PMC Journal & "helps explain why our belief in clinical effectiveness may not jibe with the research" \\
\hline $\begin{array}{l}\text { Henderson } \\
\text { CN, et al [66] }\end{array}$ & 1 & 13 & $\begin{array}{l}\text { Purchase } \\
\text { Un/author }\end{array}$ & "establishes animal model for studying mechanisms of action of spinal manipulation" \\
\hline $\begin{array}{l}\text { McGinn T, } \\
\text { et al }[67]\end{array}$ & 1 & 11 & PMC & "teaches CPRs in applying evidence" \\
\hline $\begin{array}{l}\text { Cramer GD, } \\
\text { et al [68] }\end{array}$ & 1 & 10 & Purchase & "mechanism of action of spinal manipulation" \\
\hline $\begin{array}{l}\text { Chevan J, } \\
\text { et al [69] }\end{array}$ & 1 & 8 & Journal & $\begin{array}{l}\text { "This article written by PTs cites survey research showing that more LBP patients seek DC } \\
\text { services (28\%) compared to PT services (11\%). Their discussion about the disparities between } \\
\text { DCs and PTs is quite revealing." }\end{array}$ \\
\hline $\begin{array}{l}\text { Villanueva- } \\
\text { Russell Y [14] }\end{array}$ & 1 & 6 & Purchase & "helps create professional identity" \\
\hline Bogduk N [70] & 1 & 3 & $\begin{array}{l}\text { No online } \\
\text { access }\end{array}$ & $\begin{array}{l}\text { "This article provides the reader with the [simple] tools to determine if a manuscript } \\
\text { provides the necessary information to identify the reliability and validity of diagnostic tests. } \\
\text { Chiropractors often adopt tests that they do not determine these characteristics for and } \\
\text { need to know how to do this..." }\end{array}$ \\
\hline $\begin{array}{l}\text { Haneline M } \\
{[71]}\end{array}$ & 1 & 0 & Purchase & "informs the practitioner of what is and what is not evidence-based practice" \\
\hline
\end{tabular}

PMC $=$ Article available through open access on PubMed Central. Journal = Article available through open access on journal homepage. Purchase $=$ Article available for purchase from journal. Un/Author = Unauthorized posting on Internet website.

decision-making for the care of patients with low back pain $[40-42,50,59,72]$, while two texts highlight treatment approaches to neck pain and related conditions $[45,55]$. Five articles report findings from clinical trials of manual therapies or spinal manipulation $[43,46,53,57,60]$, while three other papers discuss either ineffective applications, $[47,65]$ or common side effects of manipulation [44]. Lastly, nine articles demonstrate chiropractic research priorities [30,73,74], analyze health policy issues for the chiropractic profession $[14,51,69]$, or discuss evidence-based practice concepts for doctors and students of chiropractic [67,70,71]. That only one respondent mentioned each of these studies should not undermine their potential relevance to the chiropractic profession. Instead, many unduplicated references are important references on key concepts, evidence or issues for practicing doctors of chiropractic. For example, 10 recommended articles provide theoretical rationale or basic science evidence on the mechanisms of action of spinal or joint manipulation $[48,52,56,58,61-64,66,68]$.

\section{Scholarly impact}

The secondary analysis of the scholarly impact of the recommended PEs revealed interesting patterns that may further assist doctors of chiropractic in selecting from among this list of essential literature for the profession (Table 2). The Google Scholar citation analysis 
identified some articles as highly referenced within the field of chiropractic and in associated disciplines. Researchers have cited some chiropractic research articles more than 100 times including work by Senstad et al. [44], Hurwitz et al. [45], Pickar [36], Cassidy et al. [37], Nelson et al. [46], Bronfort et al. [35] and Olafsdottir and colleagues [47]. Several articles in fields related to chiropractic, specifically regarding the interdisciplinary care of persons with back pain, also demonstrated very high citation rates [40-43]. These articles have clearly influenced how other researchers view the field. Other articles recommended as essential literature have received much less attention from researchers. This analysis of scholarly impact does not necessarily indicate the possible influence of any article among chiropractic educators, clinicians or students.

\section{Article accessibility}

The accessibility of scholarly articles also is an important factor for the dissemination, uptake, and clinical application of scientific knowledge. Many of the most highly cited articles were freely available as full-text articles on the Internet, either from the journal publisher or through the U.S. National Library of Medicine (NLM) and its PubMed Central (PMC) database of biomedical and life sciences literatures. For example, the articles with the broadest uptake by other authors were the low back pain clinical practice guidelines and the clinical prediction rule for identifying responders to spinal manipulation, three freely accessible articles published in Annals of Internal Medicine (Table 2). In contrast, articles published in Spine, The Spine Journal, and Journal of Manipulative and Physiologic Therapeutics were available only by subscription or direct purchase, at a cost of about \$30 US per article. Perhaps not surprisingly, these articles also had lower overall citation rates from papers published in open access journals or those available from PMC. Of note, several of these articles were available on websites from individuals or institutions in possible violation of article copyright agreements.

\section{Discussion}

This survey of chiropractic research leaders sought to identify essential literature that every doctor of chiropractic and chiropractic student should read and reference to inform evidence-based clinical practice. Survey respondents identified 41 unique articles or books they considered key readings within the field of chiropractic or related disciplines. Essential literature included basic science and clinical research, health policy statements, education-based articles, and other types of evidence for the chiropractic profession. The majority of the recommended articles $(n=34)$ were published in the past 10 years, with no citations prior to 1995 offered by respondents. Two or more respondents recommended six journal articles as key pieces of evidence for doctors of chiropractic and chiropractic students [8,35-39]. These articles offered evidence on the effectiveness of manual therapies [35,38], the physiological underpinnings of spinal manipulation [36,39], risks related to chiropractic care [37], and arguments for an expanded role for chiropractors within the health care system [8], all timely and important topics for the chiropractic profession. Thirty-four additional citations on a topics ranging from low back pain and neck pain, chiropractic side effects, biomechanical and physiological effects of chiropractic adjustments, research priorities, costs and access for chiropractic therapy, and evidence-based clinical practice also were identified.

Many articles selected by respondents as essential literature had achieved some degree of scholarly impact in that they were referenced by many researchers in multiple publications $[35-37,40-42,46,49,50,53,54]$. While the selection of scholarly literature is dependent upon the specific goals and interests of the reader, an argument is made that articles cited more often both within the profession and in disciplines with a shared scope are more essential for the chiropractic professional than articles not as widely referenced. A shared knowledge base will assist doctors of chiropractic to communicate with one another, our patients, and other healthcare professionals about the evidence underpinning various treatment approaches.

The scholarly impact and clinical importance of this essential literature for the chiropractic profession also may be influenced by the access clinicians have to scientific articles [25,32-34]. Our secondary analysis found that articles published in open access journals generally had higher citation rates than journals where article access was limited to subscribers or by purchase. Clinicians may be prevented from accessing articles recommended as essential literature by costly fees, a concern identified in previous research [75]. Editors and publishers of chiropractic and spine-related journals may wish to reconsider their access policies in order to increase use of their articles by researchers and practicing clinicians.

\section{Limitations}

Extremely low response rate, high rate of attrition, coverage and non-response survey errors, and selfreport bias are limitations of this study. This extremely low response rate and high attrition rate were problematic. While 43 of 68 potential respondents agreed to participate, only $25 \%$ completed the survey. Of these 17 respondents, eight contributed only one piece of evidence, six contributed 2-5 references, and only three contributed more than five citations. This response rate is low by survey standards [76], including among surveys 
of doctors of chiropractic, which average about 53\% for postal surveys [77]. The reasons for the sharp decline between respondents agreeing to participate in the study and their actual participation are unknown. While only four e-mails were returned undeliverable, it is unknown how many potential respondents may have not have received the initial e-mail due to spam filters. Future studies could be designed using a multi-modal approach. The attrition rate was high as respondents could re-enter the survey at any time during deployment if they left the session after agreeing to participate. Both of these issues potentially affect the quality of data, specifically coverage and non-response error. Coverage and non-response errors result from all members of a population not having a known, nonzero chance of being included in the sample and by non-respondents potentially differing from respondents [76]. The high attrition, both in terms of starting the survey and stopping after submitting only one recommendation might be attributed to recall bias and or source amnesia (e.g., inability to remember where, when or how one has learned prior information while retaining its factual knowledge) [78]. Respondents also performed mental work to complete this short-answer survey rather than answering discrete categorical questions, which also can result in increased attrition [76]. And yet, this survey was web-based. Respondents were not asked to recall references from memory nor were they restricted from using on-line databases to identify essential literature, which might suggest either low familiarity with the chiropractic literature or a disinterest in the survey topic among respondents. Varying degrees of self-report bias also are possible. Respondents may have entered socially desired responses (i.e., often-cited references or citations from well-known researchers) or responses that might benefit themselves or their colleagues (i.e., referencing articles that either they or their colleagues have published). An additional limitation is the potential geographic bias in the survey as the potential respondents for this study were recruited from North American institutions. Future surveys should include chiropractic colleges and programs internationally. It is suspected that the philosophy and scope of chiropractic education, research, and practice differs between regions and, consequently, affect (or enhance) survey responses. Lastly, in such a rapidly progressing subject, new impactful articles have undoubtedly been published that should be included as "essential," prior to actual publication of this study. We should disseminate thoroughly yet quickly when compiling and distributing future study results. In spite of these limitations, we consider these responses a fruitful start to this initial investigation into a previously unexplored subject, essential literature for the chiropractic profession.

\section{Relevance/future research}

Now that a preliminary list of essential literature for doctors of chiropractic exists, a future researcher may consider surveying practicing doctors of chiropractic or chiropractic students and faculty to determine their awareness of this essential literature and to expand the key articles from the perspective of non-researchers.

\section{Conclusions}

This survey of chiropractic research leaders resulted in a manageable list of essential literature for chiropractic students and practitioners. A variety of perspectives and values are evident when looking at the outcomes of the survey. The recommended literature might be broken down into three major categories:

1 Foundational understanding of the pathophysiology underlying chiropractic concepts and practices.

2 Importance of practitioner awareness of the state of the evidence for patient care and clinical practice.

3 Potential societal impact fostering improved integration or acceptance.

The actual impact is limited to the perspectives of the responding research leaders. Regular annual or biannual surveys could be of benefit to many in the profession.

\section{Abbreviations}

EBCP: Evidence-based clinical practice; PE: Pieces of evidence; DC: Doctor of chiropractic; NLM: United States National Library of Medicine; PMC: PubMed Central.

\section{Competing interests}

The authors declare that this paper is original and has not been published nor is being considered elsewhere for publication. The authors, individually and collectively, declare no conflicts of interests.

\section{Authors' contributions}

The corresponding author, BM, planned the design, was involved in supervision, data collection/processing, analysis/interpretation, literature search, writing, and critical review of this manuscript. JS was responsible for concept development, supervision, writing, and critical review. DD was integral for design, supervision, data collection, and writing. RB was involved in writing and supervision. SS completed the secondary analysis and assisted in interpretation, writing, and critical review of this manuscript. All authors read and approved the final manuscript.

\section{Authors' information}

Barbara A. Mansholt, D.C., M.S., Assistant Professor, Technique Department, Palmer College of Chiropractic, 1000 Brady St., Davenport, IA 52803, USA. John S. Stites, D.C., Director, Community Clinics, Palmer College of Chiropractic, 2001 52nd Ave., Moline, IL 61265, USA.

Dustin C. Derby, Ed.D., Senior Director, Institutional Planning \& Research, Palmer College of Chiropractic, 723 Brady St., Davenport, IA 52803, USA. Ronald J. Boesch, D.C., Dean of Clinics, Palmer Davenport Clinical Affairs, Palmer College of Chiropractic, 1000 Brady St., Davenport, IA 52803, USA. Stacie A. Salsbury, Ph.D., R.N., Clinical Project Manager, Palmer Center for Chiropractic Research, Palmer College of Chiropractic, 741 Brady St.,

Davenport, IA 52803, USA.

\section{Author details}

${ }^{1}$ Technique Department, Palmer College of Chiropractic, 1000 Brady St, Davenport, IA 52803, USA. ${ }^{2}$ Community Clinics, Palmer College of 
Chiropractic, 2001 52nd Ave, Moline, IL 61265, USA. ${ }^{3}$ Institutional Planning \& Research, Palmer College of Chiropractic, 723 Brady St, Davenport, IA 52803, USA. ${ }^{4}$ Palmer Davenport Clinical Affairs, Palmer College of Chiropractic, 1000 Brady St, Davenport, IA 52803, USA. ${ }^{5}$ Palmer Center for Chiropractic Research, Palmer College of Chiropractic, 741 Brady St, Davenport, IA 52803, USA.

Received: 12 June 2013 Accepted: 20 September 2013

Published: 27 September 2013

\section{References}

1. Sackett DL, Rosenberg WM, Gray JA, Haynes RB, Richardson WS: Evidence based medicine: what it is and what it isn't. BMJ 1996, 312:71-72.

2. Satterfield JM, Spring B, Brownson RC, Mullen EJ, Newhouse RP, Walker BB, Whitlock EP: Toward a transdisciplinary model of evidence-based practice. Milbank Q 2009, 87:368-390.

3. Giordano J, Garcia MK, Boatwright D, Klein K: Complementary and alternative medicine in mainstream public health: a role for research in fostering integration. J Altern Complement Med 2003, 9:441-445.

4. Coulter ID, Hurwitz EL, Adams AH, Genovese BJ, Hays R, Shekelle PG: Patients using chiropractors in North America: who are they, and why are they in chiropractic care? Spine (Phila Pa 1976) 2002, 27:291-296.

5. Davis MA, Sirovich BE, Weeks WB: Utilization and expenditures on chiropractic in the United States from 1997 to 2006. Health Serv Res 2010, 45:748-761.

6. Cooper RA, McKee HJ: Chiropractic in the United States: trends and issues. Milbank Q 2003, 81:107-138.

7. Meeker WC, Haldeman S: Chiropractic: a profession at the crossroads of mainstream and alternative medicine. Ann Intern Med 2002, 136:216-227.

8. Murphy D, Justice B, Paskowski I, Perle S, Schneider M: The establishment of a primary spine care practitioner and its benefits to health care reform in the United States. Chiropr Man Therap 2011, 19:17.

9. Murphy DR, Schneider MJ, Seaman DR, Perle SM, Nelson CF: How can chiropractic become a respected mainstream profession? The example of podiatry. Chiropr Osteopat 2008, 16:10.

10. Myburgh C, Hartvigsen J, Grunnet-Nilsson N: Secondary legitimacy: a key mainstream health care inclusion strategy for the danish chiropractic profession? J Manipulative Physiol Ther 2008, 31:392-395.

11. Redwood D, Hawk C, Cambron J, Vinjamury SP, Bedard J: Do chiropractors identify with complementary and alternative medicine? Results of a survey. J Altern Complement Med 2008, 14:361-368.

12. Busse JW, Jacobs C, Ngo T, Rodine R, Torrance D, Jim J, Kulkarni AV, Petrisor B, Drew B, Bhandari M: Attitudes toward chiropractic: a survey of North American orthopedic surgeons. Spine (Phila Pa 1976) 2009, 34:2818-2825.

13. Meeker WC: Public demand and the integration of complementary and alternative medicine in the US health care system. J Manipulative Physiol Ther 2000, 23:123-126.

14. Villanueva-Russell Y: Caught in the crosshairs: Identity and cultural authority within chiropractic. Soc Sci Med 2011, 72:1826-1837.

15. Reggars J: Chiropractic at the crossroads or are we just going around in circles? Chiropr Man Therap 2011, 19:11.

16. Hunnisett $A$, Cunliffe $C$ : Chiropractic treatment as a primary care intervention for better musculoskeletal health in the aging population in the United Kingdom: an opinion and positioning paper. Front Physiol 2012, 3:87.

17. LeFebvre R, Peterson D, Haas M: Evidence-based practice and chiropractic care. JEBCAM 2013, 18(1):75-79.

18. Kreitzer MJ, Sierpina VS: NCCAM awards grants to CAM institutions to enhance research education. EXPLORE 2008, 4(1):74-76.

19. Haas M, Leo M, Peterson D, LeFebvre R, Vavrek D: Evaluation of the effects of an evidence-based practice curriculum on knowledge, attitudes, and self-assessed skills and behaviors in chiropractic students. J Manipulative Physiol Ther 2012, 35(9):701-709.

20. Harvey PJ, Goodell KJ: Development and evolution of an information literacy course for a doctor of chiropractic program. Commun Info Lit 2008, 2(1):52-61.

21. Shreeve MW: Evidence-based chiropractic education: are we equipping graduates for clinical practice with improved patient outcomes? J Chiropr Educ 2012, 26(2):184

22. Banzai R, Derby DC, Long CR, Hondras MA: International web survey of chiropractic students about evidence-based practice: a pilot study. Chiropr Man Therap 2011, 19(1):6.
23. Hall G: Attitudes of chiropractors to evidence-based practice and how this compares to other healthcare professionals: a qualitative study. Clin Chiropr 2011, 14(3):106-111.

24. Suter E, Vanderheyden LC, Trojan LS, Verhoef MJ, Armitage GD: How important is research-based practice to chiropractors and massage therapists? J Manipulative Physiol Ther 2007, 30(2):109-115.

25. Hadley J, Hassan I, Khan K: Knowledge and beliefs concerning evidence-based practice amongst complementary and alternative medicine health care practitioners and allied health care professionals: a questionnaire survey. BMC Complement Altern Med 2008, 8(1):45.

26. Maylahn C, Fleming D, Birkhead G: Health departments in a brave new world. Prev Chronic Dis 2013. 10:130003:DOI: http://dx.doi.org/10.5888/ pcd10.130003.

27. Hawk C, Meeker W, Hansen D: The national workshop to develop the chiropractic research agenda. J Manipulative Physiol Ther 1997, 20(3):147.

28. Lawrence DJ, Meeker WC, Commentary: The national workshop to develop the chiropractic research agenda: 10 years on, a new set of white papers. J Manipulative Physiol Ther 2006, 29(9):690-694.

29. Mootz RD, Hansen DT, Breen A, Killinger LZ, Nelson C: Health services research related to chiropractic: review and recommendations for research prioritization by the chiropractic profession. J Manipulative Physiol Ther 2006, 29(9):707-725.

30. Haas M, Bronfort G, Evans RL: Chiropractic clinical research: progress and recommendations. J Manipulative Physiol Ther 2006, 29(9):695-706.

31. Bogdan RCBS: Qualitative Research for Education: An Introduction to Theories and Methods. 4th edition. New York: Pearson Education Group, Inc.; 2003.

32. Leach MJ, Gillham D: Are complementary medicine practitioners implementing evidence based practice? Complement Ther Med 2011, 19(3):128-136

33. Cadmus E, Van Wynen EA, Chamberlain B, Steingall P, Kilgallen ME, Holly C Gallagher-Ford L: Nurses' skill level and access to evidence-based practice. J Nurs Adm 2008, 38(11):494-503.

34. Green ML, Ruff TR: Why do residents fail to answer their clinical questions? A qualitative study of barriers to practicing evidence-based medicine. Acad Med 2005, 80(2):176-182.

35. Bronfort G, Haas M, Evans R, Leininger B, Triano J: Effectiveness of manual therapies: the UK evidence report. Chiropr Osteopat 2010, 18:3.

36. Pickar JG: Neurophysiological effects of spinal manipulation. Spine J 2002, 2(5):357-371

37. Cassidy JD, Boyle E, Cote P, He Y, Hogg-Johnson S, Silver FL, Bondy SJ: Risk of vertebrobasilar stroke and chiropractic care: results of a populationbased case-control and case-crossover study. I Manipulative Physiol Ther 2009, 32(2 Suppl):S201-S208.

38. Walker BF, French SD, Grant W, Green S: Combined chiropractic interventions for low-back pain. Cochrane Database Syst Rev 2010, 4, CD005427.

39. Ianuzzi A, Khalsa PS: Comparison of human lumbar facet joint capsule strains during simulated high-velocity, low-amplitude spinal manipulation versus physiological motions. Spine J 2005, 5(3):277-290.

40. Chou R, Qaseem A, Snow V, Casey D, Cross JT Jr, Shekelle P, Owens DK: Diagnosis and treatment of low back pain: a joint clinical practice guideline from the American College of Physicians and the American Pain Society. Ann Intern Med 2007, 147:478-491.

41. Childs JD, Fritz JM, Flynn TW, Irrgang JJ, Johnson KK, Majkowski GR, Delitto A: A clinical prediction rule to identify patients with low back pain most likely to benefit from spinal manipulation: a validation study. Ann Int Med 2004, 141:920-928.

42. Chou R, Huffman LH: Nonpharmacologic therapies for acute and chronic low back pain: a review of the evidence for an american pain society/ american college of physicians clinical practice guideline. Ann Intern Med 2007, 147(7):492-504.

43. UK BEAM Trial Team: United Kingdom back pain exercise and manipulation (UK BEAM) randomised trial: effectiveness of physical treatments for back pain in primary care. BMJ 2004, 329(7479):1377.

44. Senstad O, Leboeuf-Yde C, Borchgrevink C: Frequency and characteristics of side effects of spinal manipulative therapy. Spine 1997, 22:435-441.

45. Hurwitz EL, Carragee EJ, van der Velde G, Carroll LJ, Nordin M, Guzman J, Peloso PM, Holm LW, Cote P, Hogg-Johnson S: Treatment of neck pain: noninvasive interventions: results of the bone and joint decade 2000-2010 task force on neck pain and its associated disorders. Spine 2008, 33(4 Suppl):S123-S152. 
46. Nelson CF, Bronfort G, Evans R, Boline P, Goldsmith C, Anderson AV: The efficacy of spinal manipulation, amitriptyline and the combination of both therapies for the prophylaxis of migraine headache. J Manipulative Physiol Ther 1998, 21(8):511-519.

47. Olafsdottir E, Forshei S, Fluge G, Markestad T: Randomised controlled trial of infantile colic treated with chiropractic spinal manipulation. Arch Dis Child 2001, 84(2):138-141.

48. Skyba DA, Radhakrishnan R, Rohlwing JJ, Wright A, Sluka KA: Joint manipulation reduces hyperalgesia by activation of monoamine receptors but not opioid or GABA receptors in the spinal cord. Pain 2003, 106(1-2):159-168.

49. Haldeman S, Carroll L, Cassidy JD, Schubert J, Nygren A: The bone and joint decade 2000-2010 task force on neck pain and its associated disorders: executive summary. Spine (Phila Pa 1976) 2008, 33(4 Suppl):S5-S7.

50. Dagenais S, Tricco AC, Haldeman S: Synthesis of recommendations for the assessment and management of low back pain from recent clinical practice guidelines. Spine J 2010, 10(6):514-529.

51. Legorreta AP, Metz RD, Nelson CF, Ray S, Chernicoff HO, Dinubile NA: Comparative analysis of individuals with and without chiropractic coverage: patient characteristics, utilization, and costs. Arch Intern Med 2004, 164(18):1985-1992.

52. Cramer GD, Gregerson DM, Knudsen JT, Hubbard BB, Ustas LM, Cantu JA The effects of side-posture positioning and spinal adjusting on the lumbar Z joints: a randomized controlled trial with sixty-four subjects. Spine 2002, 27(22):2459-2466.

53. Haas M, Groupp E, Kraemer DF: Dose-response for chiropractic care of chronic low back pain. Spine J 2004, 4(5):574-583.

54. Carroll $L$, et al: Research priorities and methodological implications: the bone and joint decade 2000-2010 task force on neck pain and its associated disorders. Spine (Phila Pa.1976) 2008, 33(4 Suppl):S214-S220.

55. Bogduk N, McGuirk B: Management of Acute and Chronic Neck Pain: An Evidence-Based Approach. Philadelphia, PA: Elsevier; 2006.

56. Pickar JG, Kang YM: Paraspinal muscle spindle responses to the duration of a spinal manipulation under force control. $J$ Manipulative Physiol Ther 2006, 29(1):22-31.

57. Haas M, Spegman A, Peterson D, Aickin M, Vavrek D: Dose response and efficacy of spinal manipulation for chronic cervicogenic headache: a pilot randomized controlled trial. Spine J 2010, 10(2):117-128.

58. Reggars J, Pollard HP: Analysis of zygapophyseal joint cracking during chiropractic manipulation. J Manipulative Physiol Ther 1995, 18(2):65-71.

59. Murphy DR: A theoretical model for the development of a diagnosis-based clinical decision rule for the management of patients with spinal pain. BMC Musculoskelet Disord 2007, 8:75.

60. Bishop PB, Quon JA, Fisher CG, Dvorak MF: The chiropractic hospital-based interventions research outcomes (CHIRO) study: a randomized controlled trial on the effectiveness of clinical practice guidelines in the medical and chiropractic management of patients with acute mechanical low back pain. Spine J 2010, 10(12):1055-1064.

61. Little JS, lanuzzi A, Chiu JB, Baitner A, Khalsa PS: Human lumbar facet joint capsule strains: II. Alteration of strains subsequent to anterior interbody fixation. Spine J 2004, 4(2):153-162.

62. Pickar JG, Sung PS, Kang YM, Ge W: Response of lumbar paraspinal muscles spindles is greater to spinal manipulative loading compared with slower loading under length control. Spine J 2007, 7(5):583-595.

63. Herzog W: The biomechanics of spinal manipulation. J Bodyw Mov Ther 2010, 14(3):280-286.

64. lanuzzi A, Khalsa PS: High loading rate during spinal manipulation produces unique facet joint capsule strain patterns compared with axial rotations. J Manipulative Physiol Ther 2005, 28(9):673-687.

65. Hartman S: Why do ineffective treatments seem helpful? A brief review. Chiropr Osteo 2009, 17(1):10.

66. Henderson CN, Cramer GD, Zhang Q, Devocht JW, Fournier JT: Introducing the external link model for studying spine fixation and misalignment: part 1-need, rationale, and applications. J Manipulative Physiol Ther 2007, 30(3):239-245.

67. McGinn T, Jervis R, Wisnivesky J, Keitz S, Wyer P: Tips for teachers of evidence-based medicine: clinical prediction rules (CPRs) and estimating pretest probability. J Gen Intern Med 2008, 23(8):1261-1268

68. Cramer GD, Henderson CNR, Little JW, Daley C, Grieve TJ: Zygapophyseal joint adhesions after induced hypomobility. J Manipulative Physiol Ther 2010, 33:508-518.
69. Chevan J, Riddle DL: Factors associated with care seeking from physicians, physical therapists, or chiropractors by persons with spinal pain: a population-based study. J Orthop Sports Phys Ther 2011, 41(7):467.

70. Bogduk N: Truth in musculoskeletal medicine: truth in diagnosis - validity. Austr J Musculoskeletal Med 1999, 4:32-39.

71. Haneline M: A primer on evidence-based practice for chiropractors. Chiropr J Aust 2011, 41(3):78.

72. Murphy D, Hurwitz E, Nelson C: A diagnosis-based clinical decision rule for spinal pain part 2: review of the literature. Chiropr Osteopat 2008, 16(1):7.

73. Haldeman S, Carroll L, Cassidy JD, Schubert J, Nygren A: The bone and joint decade 2000-2010 task force on neck pain and its associated disorders. Eur Spine J 2008, 17(1):5-7.

74. Carroll L, Hurwitz E, Cote P, Hogg-Johnson S, Carragee E, Nordin M, Holm L, Velde G, Cassidy JD, Guzman J, Peloso P, Haldeman S: Research priorities and methodological implications. Eur Spine J 2008, 17(1):214-220.

75. Willinsky J, Quint-Rapoport M: How Complementary and Alternative Medicine Practitioners use PubMed. Internet Res: J Medical; 2007:9(2).

76. Dillman DA, Smyth JD, Christian LM: Internet, Mail, and Mixed-Mode Surveys: The Tailored Design Method. Hoboken: NJ: Wiley \& Sons; 2009.

77. Russell ML, Verhoef MJ, Injeyan HS, McMorland DG: Response rates for surveys of chiropractors. J Manipulative Physiol Ther 2004, 27:43-48.

78. Schacter DL, Harbluk JL, McLachlan DR: Retrieval without recollection: an experimental analysis of source amnesia. J Verbal Learning Verbal Behav 1984, 23(5):593-611.

doi:10.1186/2045-709X-21-33

Cite this article as: Mansholt et al:: Essential literature for the chiropractic profession: a survey of chiropractic research leaders. Chiropractic \& Manual Therapies 2013 21:33.

\section{Submit your next manuscript to BioMed Central and take full advantage of:}

- Convenient online submission

- Thorough peer review

- No space constraints or color figure charges

- Immediate publication on acceptance

- Inclusion in PubMed, CAS, Scopus and Google Scholar

- Research which is freely available for redistribution 